\title{
Diffuse Neurofibroma: An Uncommon Cause of Alopecia
}

\author{
I-Ting Lee Jian-Min Chang Yu Fu \\ Department of Dermatology, Beijing Hospital, National Center of Gerontology, Beijing, China
}

\section{Novel Insights}

- Skin biopsy showed neurofibroma (NF) occupying the entire dermis, and "pushing" hair follicles towards the upper dermis may be a clue to alopecia.

\section{Established Facts}

- Diffuse NF is a rare form of NF reported to occur primarily in children and young adults. It generally presents as a variably sized area of marked dermal and subcutaneous thickening, most often in the head and neck regions.

- The mechanism of NF-associated alopecia is unknown.

\section{Keywords}

Alopecia $\cdot$ Neurofibroma $\cdot$ Scalp

\begin{abstract}
Alopecia (hair loss) is reported to be associated with infection, genetics, hormonal changes, drugs, or inflammation. The most frequent causes of hair loss in pediatric patients include tinea capitis, alopecia areata, traction alopecia, and trichotillomania. In the adult population, causes to be considered are alopecia areata and hair loss associated with systemic disease and hormonal influence. The clinician must be able to separate the types and causes of hair loss into those that reflect primary dermatologic conditions and those that represent a reaction to systemic disease. Benign cutaneous tumors have also been known to develop alopecia via occupying the place for hair follicles or tumor-in-
\end{abstract}

duced inflammatory responses. However, the mechanism of alopecia by neurofibroma (NF) has not been well investigated. We present a 32-year-old patient who developed scalp plaque with alopecia within 6 years. He denies any itching or pain in the area, and has not tried any treatments. In this study, the unusual association between NF and alopecia is shown.

(c) 2020 S. Karger AG, Basel

\section{Introduction}

Schwannomas and neurofibromas (NF) are the most common benign tumors of the nerve sheath. The latter represents approximately 5\% of all benign soft-tissue tumors in large surgical series and is usually divided in three different types: localized, plexiform, and diffuse. Diffuse karger@karger.com www.karger.com/sad

Karger"

\section{(C) 2020 S. Karger AG, Base}

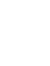




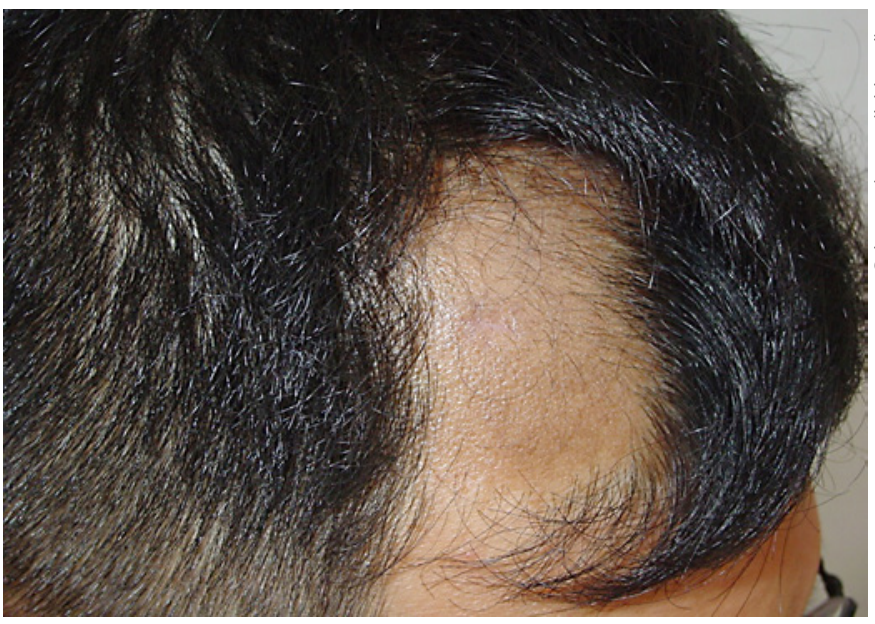

Fig. 1. Clinical image of bald patches on the patient's scalp.

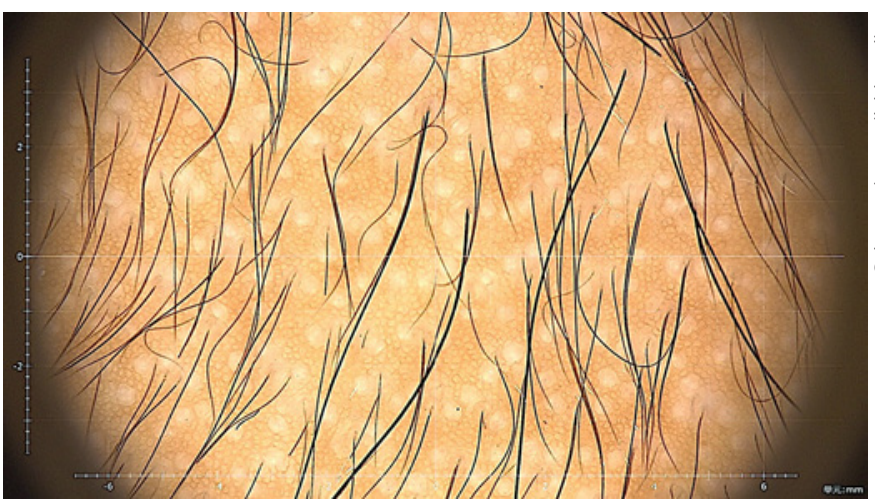

Fig. 2. Dermatoscopy shows regularly distributed small pinpoint white dots and numerous short vellus hairs.

$\mathrm{NF}$ is a rare form of NF reported to occur primarily in children and young adults. It generally presents as a variably sized area of marked dermal and subcutaneous thickening, most often in the head and neck regions $[1,2]$. However, there are only a few case reports on NF presenting as alopecic plaques. We report a case of a NF on the scalp of a 32-year-old man, which was confirmed by histopathologic examination. This case is reported for its rarity and its unique presentation.

\section{Case Report}

A 32-year-old man was seen at our outpatient clinic because of a plaque with loss of overlying hair on the anterior aspect of his scalp. The mass was first observed at the age of 26 years, and

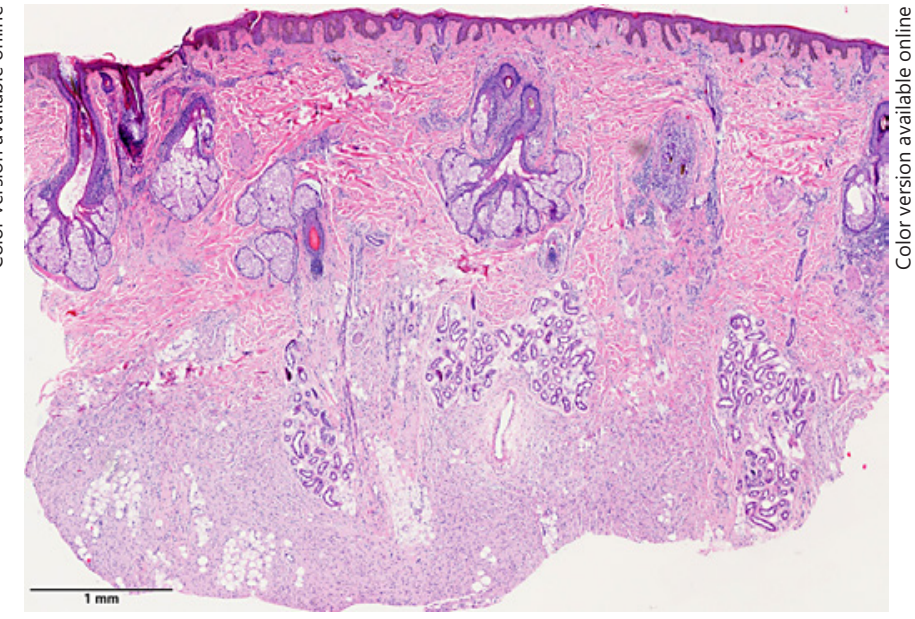

Fig. 3. The biopsy specimen of the tumor showed an infiltrative lesion occupying the dermis and subcutaneous tissue.

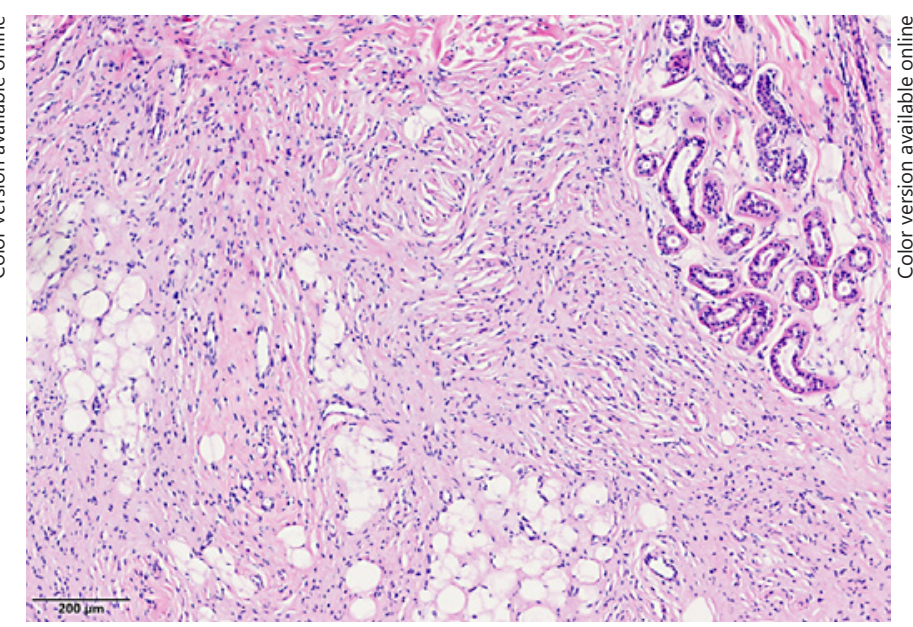

Fig. 4. Few ecstatic blood vessels were also found.

it had slowly increased in size since then. Physical examination revealed a soft $6 \times 4 \mathrm{~cm}$ skin colored mass on the anterior scalp (Fig. 1). Dermatoscopy showed regularly distributed small pinpoint white dots and numerous thin pigmented linear short hairs (Fig. 2). The rest of the cutaneous examination was unremarkable and failed to reveal any cafe-au-lait spots or axillary/ inguinal freckling. At the time, the tentative diagnosis is alopecia areata. But histologic examination of the excised specimen (Fig. 3) showed a typical presentation of diffuse NF involving the dermis and subcutaneous tissue. The lesion was characterized by a proliferation of spindle cells that contained elongated ovoid to curved nuclei, and these cells were surrounded by a matrix with wire-like collagenous fibers. Few ecstatic blood vessels were also found (Fig. 4). Then, IHC for S-100 was done, which showed strong and diffuse S-100 positivity in NF-like component (Fig. 5). 


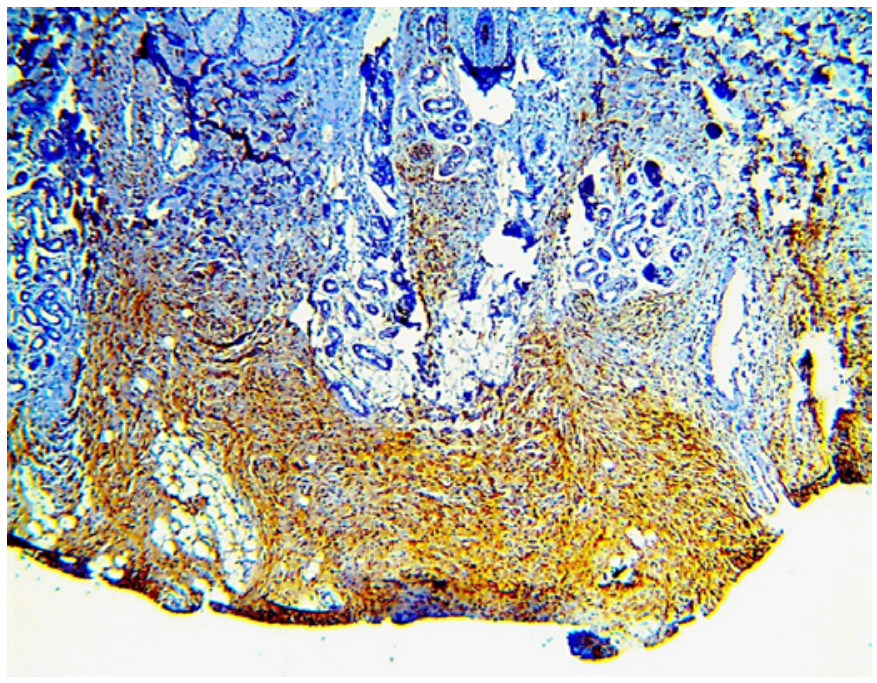

Fig. 5. IHC showing variable S-100 positivity in the NF area.

\section{Discussion}

NFs are benign tumors of the peripheral nerves that have a neuroectodermal origin [3]. Diffuse NFs are a rare subtype. They are usually single lesions, with progressive enlargement and infiltrative growth pattern, simulating malignant tumors. Intracranial extension of the extracranial variety of this tumor has rarely been described [4]. Although Kumar et al. [5] reported higher association rates, it is estimated that about $10 \%$ of these tumors are associated with NF-1. On histopathological examination, diffuse NFs are ill-defined tumors that diffusely infiltrate the dermis and subcutaneous tissue, often extending along connective tissue septa, without destroying the normal structures. They are composed of elongated spindle-shaped cells with round to fusiform nuclei and eosinophilic cytoplasm within a loose matrix of fibrillary collagen. Meissner bodies, a characteristic feature of diffuse NFs, are not always present. Fat and ectatic blood vessels may be dominant features. Expression of S-100 protein is characteristic and a sensitive, but nonspecific marker. Since malignant spindle-cell neoplasms can occur on the scalp, sarcomas, particularly dermatofibrosarcoma protuberans and desmoplastic melanoma (DM), should be ruled out. Dermatofibrosarcoma protuberans is a rare, locally invasive malignant tumor with a fibroblastic differentiation and an infrequent propensity to metastatic spread. It usually appears as a slow-growing, skin-colored to violaceous single lesion involving an extremity, consisting of a proliferation of CD34-positive spindle cells with a storiform/fascicular pattern involving the dermis and the subcutis. DM is a rare variant of spindle- cell malignant melanoma. It is easily misdiagnosed at an early stage because it can be confused with benign entities. Histological analysis, including careful attention to the presence of atypical spindle cells, as well as to lymphocytic aggregates in an abundant fibrotic stroma in the dermis, provides clues for diagnosis [6].

Currently, surgical excision is the best treatment option for isolated NFs, especially for large, painful, or functioncompromising lesions. Given the infiltrative growth pattern and multicentricity of the tumor, clinical recurrences are common, even after complete surgical excision. Because of the recurrence potential, malignant transformation, and NF development possibility, yearly follow-up is recommended [7]. Due to the lack of distinctive clinical characteristics, especially in isolated lesions, it is difficult to diagnose the lesion preoperatively, and such lesions remain largely a histopathologic diagnosis. Proper history-taking, physical examination, ophthalmologic and radiodiagnostic investigations are warranted in such cases, with special attention to detection of cafe-au-lait spots, Lisch nodules, and bilateral acoustic to exclude neurofibromatoses.

In the reported case, the presentation of diffuse NF as alopecic plaques on the scalp is illustrative of its indistinctive clinical characteristics. The mechanism of NF-associated alopecia is unknown. Further investigations are still needed to dissect the pathological connection between NF and alopecia. Thus, diffuse NF should always be kept in mind in the differential diagnosis of any indurated mass on the scalp.

\section{Statement of Ethics}

The patient has given his informed consent to publish the photos and details of the case.

\section{Disclosure Statement}

The authors have no conflicts of interest to disclose.

\section{Funding Sources}

There are no funding sources in this case report.

\section{Author Contributions}

Yu Fu (co-author): conceived and/or designed the work that led to the submission, acquired data, and/or played an important role in interpreting the results. I-Ting Lee (co-author): drafted or revised the manuscript. Jian-Min Chang (corresponding author): approved the final version. 


\section{References}

1 van Zuuren EJ, Posma AN. Diffuse neurofibroma on the lower back. J Am Acad Dermatol. 2003 Jun;48(6):938-40.

2 Yoo KH, Kim BJ, Rho YK, Lee JW, Kim YJ, Kim MN, et al. A case of diffuse neurofibroma of the scalp. Ann Dermatol. 2009 Feb;21(1): 46-8.

3 Coakley D, Atlas MD. Diffuse neurofibroma obstructing the external auditory meatus. J Laryngol Otol. 1997 Feb;111(2):145-7.
4 Ergün SS, Emel E, Karabekir S, Büyükbabani N. Extracranial diffuse neurofibroma with intracranial extension. Plast Reconstr Surg. 2000 Feb;105(2):801-3.

5 Kumar BS, Gopal M, Talwar A, Ramesh M. Diffuse neurofibroma of the scalp presenting as circumscribed alopecic patch. Int J Trichology. 2010 Jan;2(1):60-2.
6 Elsensohn A, Shiu J, Grove N, Hosking AM, Barr R, de Feraudy S. Distinguishing Neurofibroma From Desmoplastic Melanoma: the Value of p53 [J]. Am J Surg Pathol. 2018 Mar; 42(3):372-5.

7 Macias VC, Rafael M, Fernandes C, Rosa JC. Diffuse neurofibroma-an uncommon cause of alopecia [J]. An Bras Dermatol. 2013 NovDec;88(6 Suppl 1):166-9. 